\title{
SAE International
} COLLECTIONS

SAE Home > Collections > COLL-TP-00108 > Contents

Contents of Recent Developments in Aerospace Manufacturing, Materials, and Structures 2010 Collection Details Contents

\begin{tabular}{|c|c|}
\hline \multicolumn{2}{|c|}{ Search this Collection } \\
\hline & All \\
\hline \multicolumn{2}{|c|}{ Add Search Criteria: Another Text Field | Publication Date } \\
\hline \multicolumn{2}{|c|}{ Browse this Collection } \\
\hline Viewing 1 to 18 of 18 & Back | Nex \\
\hline $2010-01-1878$ & $\begin{array}{l}\text { An Experimental Strategy for the Manufacture of Aviation Fuel } \\
\text { Xuan Phuong, P. } \\
\text { SAE Technical Paper, } 2010 \text {, doi:10.4271/2010-01-1878 }\end{array}$ \\
\hline $2010-01-1877$ & $\begin{array}{l}\text { Production Implementation of Multiple Machine, High Speed Fiber } \\
\text { Placement for Large Structures } \\
\text { Rudberg, T., Flynn, R., and Nielson, J. } \\
\text { SAE Technical Paper, 2010, doi:10.4271/2010-01-1877 }\end{array}$ \\
\hline 2010-01-1869 & $\begin{array}{l}\text { Direct Drive Friction Welding: A Comprehensive Mathematical Model } \\
\text { Trancossi, M. and Dumas, A. } \\
\text { SAE Technical Paper, 2010, doi:10.4271/2010-01-1869 }\end{array}$ \\
\hline $2010-01-1868$ & $\begin{array}{l}\text { Tool Wear and Hole Quality in Drilling of Composite/Titanium Stacks } \\
\text { with Carbide and PCD Tools } \\
\text { Kim, D., Kwon, P., Lantrip, J., Beal, A. et al. } \\
\text { SAE Technical Paper, 2010, doi:10.4271/2010-01-1868 }\end{array}$ \\
\hline $2010-01-1867$ & $\begin{array}{l}\text { Drilling Mixed Stack Materials for the BOEING } 787 \\
\text { Whinnem, E. and Lipczynski, G. } \\
\text { SAE Technical Paper, 2010, doi:10.4271/2010-01-1867 }\end{array}$ \\
\hline $2010-01-1864$ & $\begin{array}{l}\text { The Use of 3D Laser Scanners in Statistical Process Control } \\
\text { Megahed, F., Wells, L., and Camelio, J. } \\
\text { SAE Technical Paper, 2010, doi:10.4271/2010-01-1864 }\end{array}$ \\
\hline $2010-01-1860$ & $\begin{array}{l}\text { Methods for Metrology Assisted Composite Part Production Part } 1 \\
\text { Holden, R., Brady, N., Lightowler, P., Heley, J. et al. } \\
\text { SAE Technical Paper, 2010, doi:10.4271/2010-01-1860 }\end{array}$ \\
\hline 2010-01-1859 & $\begin{array}{l}\text { Robot Integrated Metrology for Complex Part Manufacturing } \\
\text { Holden, R., Lightowler, P., and Brady, N. } \\
\text { SAE Technical Paper, 2010, doi:10.4271/2010-01-1859 }\end{array}$ \\
\hline 2010-01-1858 & $\begin{array}{l}\text { Method for Metrology Assisted Composite Part Production Part } 2 \\
\text { Holden, R., Heley, J., Mee, K., Morgan, B. et al. } \\
\text { SAE Technical Paper, 2010, doi:10.4271/2010-01-1858 }\end{array}$ \\
\hline $2010-01-1853$ & $\begin{array}{l}\text { Solution for Dry-Fiber-Placement with a Standard Articulating Robot } \\
\text { System } \\
\text { Dierssen, T., Bloedorn, C., and Mehlenhoff, T. } \\
\text { SAE Technical Paper, 2010, doi:10.4271/2010-01-1853 }\end{array}$ \\
\hline $2010-01-1848$ & $\begin{array}{l}\text { Adaptive Automated Assembly of Wing System Components } \\
\text { Webb, P., Michel, C., Kayani, A., Poad, M. et al. } \\
\text { SAE Technical Paper, 2010, doi:10.4271/2010-01-1848 }\end{array}$ \\
\hline $2010-01-1847$ & $\begin{array}{l}\text { Standard Test Protocol for Large Volume Robot Applications } \\
\text { Holden, R., Lightowler, P., Kingston, R., Heley, J. et al. } \\
\text { SAE Technical Paper, 2010, doi:10.4271/2010-01-1847 }\end{array}$ \\
\hline $2010-01-1846$ & $\begin{array}{l}\text { Expanding the Use of Robotics in Airframe Assembly Via Accurate } \\
\text { Robot Technology } \\
\text { Devlieg, R. } \\
\text { SAE Technical Paper, } 2010 \text {, doi:10.4271/2010-01-1846 }\end{array}$ \\
\hline $2010-01-1834$ & $\begin{array}{l}\text { Fabrication of Titanium Aerospace Hardware using Elevated } \\
\text { Temperature Forming Processes } \\
\text { Hefti, L. } \\
\text { SAE Technical Paper, } 2010 \text {, doi:10.4271/2010-01-1834 }\end{array}$ \\
\hline $2010-01-1833$ & $\begin{array}{l}\text { A Fastener Analysis Addressing Various Types of Misfit and an } \\
\text { Innovative Simple Design Solution } \\
\text { Anandan, V. } \\
\text { SAE Technical Paper, 2010, doi:10.4271/2010-01-1833 }\end{array}$ \\
\hline $2010-01-1832$ & $\begin{array}{l}\text { Joint Stiffness Optimization Techniques } \\
\text { Nagappan, K. } \\
\text { SAE Technical Paper, 2010, doi:10.4271/2010-01-1832 }\end{array}$ \\
\hline
\end{tabular}

${ }^{*}$ The appropriate SAE Member discount will be applied through the Shopping Cart process. Discounts vary according to level of membership: Elite Member $20 \%$

Premium Member 15\%

Classic Member $10 \%$

Information on: Copyright \& Usage 


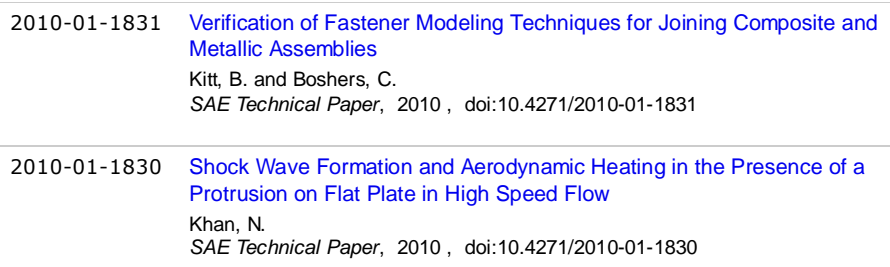

